\title{
What could we learn about high energy particle physics from cosmological observations at largest spatial scales?
}

\author{
Dmitry Gorbunov ${ }^{1, a}$ \\ ${ }^{1}$ Institute for Nuclear Research of Russian Academy of Sciences, 117312 Moscow, Russia
}

\begin{abstract}
The very well known example of cosmology testing particle physics is the number of relativistic particles (photons and three active neutrinos within the Standard Model) at primordial nucleosynthesis. These days the earliest moment we can hope to probe with present cosmological data is the early time inflation. The particle physics conditions there and now are different because of different energy scales and different values of the scalar fields, that usually prohibits a reliable connection between the particle physics parameters at the two interesting epochs. The physics at the highest energy scales may be probed with observations at the largest spatial scales (just somewhat smaller than the size of the visible Universe). However, we are not (yet) ready to make the tests realistic, because of lack of a self-consistent theoretical description of the presently favorite cosmological models to be valid right after inflation.
\end{abstract}

\section{Introduction}

The Universe is expanding, which is a most natural conclusion from observations of the Doppler redshift of the spectra emitted by distant astrophysical sources. The redshift $z$ is defined through the ratio of wavelengths of absorbed and emitted photons, respectively,

$$
\frac{\lambda_{\text {abs. }}}{\lambda_{\text {em. }}} \equiv 1+z
$$

The larger the redshift is, the larger distance has been covered by a photon and hence the earlier it has been emitted. The host of observed galaxies mostly refer to redshift $z \lesssim 4$, but there are exceptions with higher redshifts upto $z \sim 10$.

It is convenient to introduce a time-dependent scale factor $a(t)$, so that all the cosmological physical distances, like distances between galaxy clusters, grow proportionally to the scale factor, $L \propto a(t)$, while the Universe expands. The scale factor is not physical by itself: one can always rescale everything, e.g. replace meters with feet. What matters is its time-dependence, and so the physical quantity describing the expansion is the Hubble parameter,

$$
H(t) \equiv \frac{\dot{a}(t)}{a(t)},
$$

a e-mail: gorby@ms2.inr.ac.ru 
the bigger its value, the higher the Universe expansion rate is. At small redshifts, $z \ll 1$, from eqs. (1), (2) one finds the Hubble law

$$
H\left(t_{0}\right) r=z,
$$

which states that the distance to the object $r$ is proportional to its redshift $z$. The factor entering this relation, dubbed the Hubble constant, $H\left(t_{0}\right) \equiv H_{0}$, is actually the value of the Hubble parameter at present. Studying the distance-redshift relation (3) enables one to measure the present expansion rate $H_{0}$.

The Universe is homogeneous and isotropic, that we deduced from the analysis of distribution of the galaxies and galaxy clusters in the visible part of the Universe. An enormous progress here happened in the last decade thanks to spectacular performance of the astronomical telescopes deeply surveying the sky, especially Sloan Digital Sky Survey project. There were several claims of spaceanisotropy, which check failed. Admittedly, there are large voids, the hugest one is particularly interesting, but it is the only void of that scale, and may be just a fluctuation.

The Universe is occupied by "thermal" photons: cosmic microwave background (CMB) radiation with spectrum identical to that of the black body with temperature $T_{0}=2.726 \mathrm{~K}$. Both shape and normalization of the spectrum fit, the number density of the relic photons is $n_{\gamma}=411 \mathrm{~cm}^{-3}$. The spectrum has been measured over the three decades in frequency (logarithmic scale) with accuracy in temperature better than a per mille.

The widely accepted general conclusion is that our Universe is homogeneous, isotropic, hot and expanding. With expanding Euclidean manifold as a space part the interval between events gets modified as

$$
\Delta s^{2}=c^{2} \Delta t^{2}-a^{2}(t) \Delta \mathbf{x}^{2} .
$$

If we can measure how the photons propagate, we can extract $a(t)$ and thus understand the Universe expansion law. The next step is to connect it with fundamental physics through a bridge between General Relativity and particle physics. The bridge is called the Friedman equation (the first of the Einstein equations),

$$
\left(\frac{\dot{a}}{a}\right)^{2}=H^{2}(t)=\frac{8 \pi}{3} G \rho_{\text {density }}^{\text {energy }}(t),
$$

and it relates the expansion rate $H(t)$ with the total energy density of the Universe $\rho_{\text {density }}^{\text {energy }}(t)$. The higher the density is, the faster the Universe expands.

The Hubble law (3) allows for estimating the present Hubble parameter $H_{0}$, and hence the energy density of the Universe from the Friedman equation (4). To understand the content of the present Universe, one must measure the evolution of the Hubble parameter in time. The latter allows one to discriminate between different contributions because their different dependence on the scale factor: matter, radiation and curvature behave as

$$
\rho_{\text {matter }} \propto \frac{1}{a^{3}(t)}, \quad \rho_{\text {radiation }} \propto \frac{1}{a^{4}(t)}, \quad \rho_{\text {curvature }} \propto \frac{1}{a^{2}(t)},
$$

respectively. Presently dominating dark energy exhibits no noticeable (so far) dependence on the scale factor,

$$
\rho_{\text {dark energy }} \approx \text { const. }
$$

Since the Friedman equation (4) is symmetric with respect to time reversal, one concludes, that in the past the matter density was higher, our Universe was "hotter", and was filled with electromagnetic plasma. Actually, following the Friedman equation (4) and based on the cosmological observations we can confidently track the history of our Universe back to the epoch when the plasma temperature was as high as $2-3 \mathrm{MeV}$. At that epoch active neutrinos decouple from the primordial plasma 
populated by electrons, positrons, photons, protons and neutrons. As a collisionless component, neutrinos, however, proceed to contribute to the energy density and pressure and hence influence the Universe expansion. The timetable of the late Universe, with major events like primordial nucleosynthesis, the transition from radiation domination to matter domination (RD/MD equality epoch) and recombination, is determined by the Universe expansion rate in the corresponding epochs. Carefully measuring the chemical composition of the detached cold gas clouds we extract the light chemical element abundances and by comparing them with the nucleosynthesis predictions we determine the Universe expansion rate at that epoch. Then the Friedman equation (4) gives the amount of relativistic species at that time, in this way we are sure the Standard Model (SM) neutrinos have been there. The Universe population at $\mathrm{RD} / \mathrm{MD}$ and recombination we know thanks to the cosmological observations of CMB and matter distribution (galaxies, galaxy clusters) in the late Universe. Likewise, observations of quasars, supernovae type Ia, and galaxy clusters formation are used to investigate reionization and deceleration-acceleration transition.

Cosmology definitely asks for new physics, most probably new particle physics. However, it gives no any hints of the corresponding energy scales. Instead of that, cosmology indicates the time-scales associated with corresponding new physics. A physical process usually becomes of importance in the expanding Universe when its rate $\Gamma$ are of about the Universe expansion rate,

$$
\Gamma \sim H \propto \frac{T^{2}}{M_{\mathrm{Pl}}},
$$

where the Planck mass is related to the gravitational constant as $M_{\mathrm{Pl}} \equiv 1 / \sqrt{G_{N}}$. From the analysis of expanding history of the early Universe one concludes that the baryogenesis must happened some time before the temperature of primordial plasma dropped down to $1 \mathrm{MeV}$, as the matter-antimatter asymmetry has generated by the nucleosynthesis epoch. The dark matter must be produced well before the epoch of RD/MD equality, $T \sim 1 \mathrm{eV}$. The dark energy must be present by $T \simeq 5 \mathrm{~K}$. Rates $\Gamma$ of the corresponding new physics processes must exceed the expansion rates $H$ at the epochs with plasma of the corresponding temperatures $T$ in accordance with eq. (5). In this way cosmological observations constrain the new physics scales. The earliest epoch which we know about directly, from analysis of the observational data (chemical element abundances), is the pre-nucleosyntesis epoch of $T \sim 1 \mathrm{MeV}$. Was the Universe hotter we know not, at least for sure.

\section{Large scale structures, matter inhomogeneities and inflation}

However, there are observations which most probably advertise the presence of other new physics operating when the Universe was much younger, and its energy density significantly exceeding that of the pre-nucleosynthesis epoch. These observations concern the inhomogeneous Universe: CMB anisotropy and Large Scale Structure. Both are originated from the matter inhomogeneities in the early Universe. Their seeds are initial scalar perturbations of matter density with amplitude of $\delta \rho / \rho \sim \delta T / T \sim 10^{-4}$. Combined analysis of the cosmological data revealed the properties of these perturbations. They are adiabatic, so that while the total matter energy density fluctuates, the relative concentration of all species remains intact,

$$
\delta\left(\frac{n_{B}}{s}\right)=\delta\left(\frac{n_{D M}}{s}\right)=\delta\left(\frac{n_{L}}{s}\right)=0 .
$$

The initial matter inhomogeneities are Gaussian, so all the correlation functions in the Fourier space are described by only the two-point function, which behaves as

$$
\left\langle\frac{\delta \rho}{\rho}(\mathbf{k}) \frac{\delta \rho}{\rho}\left(\mathbf{k}^{\prime}\right)\right\rangle \propto\left(\frac{\delta \rho}{\rho}(\mathbf{k})\right)^{2} \times \delta\left(\mathbf{k}+\mathbf{k}^{\prime}\right) .
$$


The scalar perturbations exhibit a very flat power spectrum,

$$
\left\langle\left(\frac{\delta \rho}{\rho}(\mathbf{x})\right)^{2}\right\rangle=\int_{o}^{\infty} \frac{\mathbf{d k}}{\mathbf{k}} \mathcal{P}_{S}(\mathbf{k}), \quad \mathcal{P}_{S}(\mathbf{k}) \approx \text { const },
$$

and the combined analysis of cosmological data on $\mathrm{CMB}$ and large scale structure favors [1]

$$
\mathcal{P}_{S} \equiv A_{S} \times\left(\frac{k}{k_{*}}\right)^{n_{s}-1}, \quad A_{S} \approx 2.5 \times 10^{-9}, \quad n_{S} \approx 0.97 .
$$

There are no viable sources of the initial scalar perturbations fueling during the standard Hot Universe expansion. Most probably they emerge earlier, at the inflationary stage of almost exponential expansion specially designed to explain while the Universe is flat, homogeneous and isotropic. The exponential expansion is provided by a specific dynamics of a scalar field dubbed inflaton. At the inflationary stage it evolves very slowly, mimicking the system of constant energy density. It makes the scale factor grow exponentially, as follows from the Friedman equation (4). Quantum fluctuations of this field, as well as spin-2 quantum fluctuations of metric — so-called tensor perturbations or gravity waves - evolve very peculiarly. The amplitude of a perturbation of wave-length $\lambda \propto 1 / a$ decreases inversely proportional to the scale factor until the moment, when the 3-momentum $q=2 \pi / \lambda$ becomes of about the Hubble parameter, $q \approx H$, so-called horizon crossing. From this moment on, the wavelength proceeds to stretch but in the regime $\lambda \gg 1 / H$ (the mode is called superhorizon) the amplitude remains frozen at the value $\delta \varphi_{\lambda}=H /(2 \pi)$. The process is illustrated in Fig. 1. For the
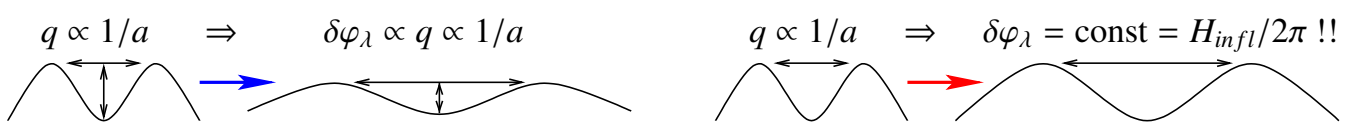

Figure 1. At inflationary stage, the amplitude of subhorizon modes, $q \gg H$, left panel, decreases as $\delta \varphi_{\lambda} \propto q$, while the mode wave-length stretches as $\lambda=2 \pi / q \propto a$; meanwhile the amplitude of superhorizon modes, $q \ll H$, right panel, remains constant at the value of Hubble parameter taken at the moment of the horizon crossing, $\delta \varphi_{\lambda}=H_{\text {infl }}(q=H) / 2 \pi$.

superhorizon mode the proper quantum amplitude must become exponentially small $\delta \varphi_{\lambda}^{\text {quantum }} \propto 1 / a$ with exponentially growing scale factor. On the contrary, the amplitude remains constant, and hence becomes populated by exponentially large number of quanta, i.e. becomes classical. This miraculous process results in production of classical spatial perturbations of inflaton field and metric.

Later, at the post-inflationary stage, reheating, and hot stages of the Universe evolution, the wavelength continues to grow proportionally to the scale factor, while the horizon $l_{H} \propto 1 / H$ grows slower and hence at some moment the mode crosses the horizon once again becoming subhorizon, see Fig. 2. Then the amplitude starts to evolve obeying the linear perturbation equations written above the cosmological background determined by the homogeneous component of the primordial plasma. Later, after $\mathrm{RD} / \mathrm{MD}$ equality, the dark matter perturbations start to dominate and finally decouple from the Hubble flow. They make the local gravitational potentials which form the proto-halos of future galaxies and galaxy clusters the ordinary matter (baryons) inhabited after recombination.

The inflaton field perturbations $\delta \phi(x)$ give birth to the inhomogeneities of the matter distribution $\delta \rho(x)$, while the metric perturbations give rise to the primordial gravitational waves $h_{\mu v}(x)$. The contrasts of the matter distribution $\delta \rho / \rho$ and amplitudes of the gravitational waves $h$ are related to the 


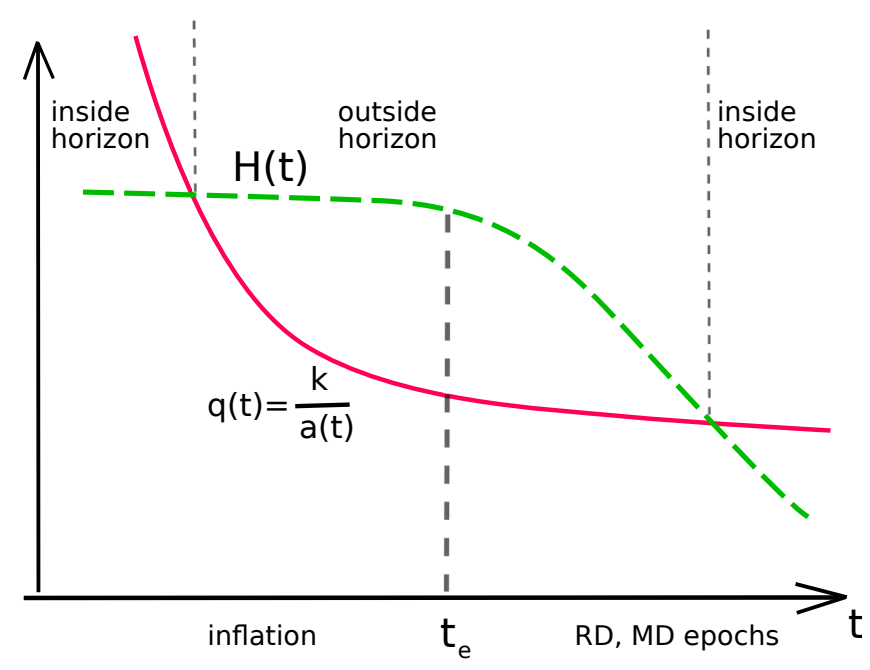

Figure 2. Evolution of the physical momentum $q=k / a(t)$ of the mode of conformal momentum $k$ and the Hubble parameter $H(t)$ in the realistic Universe. Originated from the quantum fluctuation at the inflationary stage, the mode decreases exponentially at that time until the horizon crossing, $H=q$. Then, while outside the horizon it remains constant, and starts to evolve later, only after the horizon entrance at the hot stage of the Universe expansion.

Hubble parameter, inflaton field and its potential at inflation as follows (for details see e.g. [2])

$$
\delta \phi \rightarrow \frac{\delta \rho}{\rho} \sim \frac{H^{2}}{\dot{\phi}} \propto \frac{V^{3 / 2}}{V^{\prime}}, \quad h \sim \frac{H}{M_{P l}} \propto V^{1 / 2} .
$$

From the absence of any hints of the tensor modes one places an upper limit on the Hubble parameter at inflation, $H_{\text {inf }}$, and hence at the energy scale of inflation at the level of

$$
V^{1 / 4} \lesssim 10^{16} \mathrm{GeV}
$$

Therefore, the new physics, we can hope to probe with cosmological observables sensitive to the inflationary dynamics, dwells below this scale.

One of the consequences of the absence of the relic gravity waves and the corresponding upper limit on the inflationary expansion rate is a severe constraint on the electroweak Higgs sector. In the SM the quantum corrections to the effective Higgs potential turn the Higgs self-coupling to negative values at large Higgs fields, and the SM Higgs potential gains another, global minimum in the (super)planckian region, see right panel of Fig. 3. At the inflationary stage all the fields fluctuate at $\delta \phi \sim H / 2 \pi$, including the SM Higgs. The cosmological evolution definitely must not end up with the SM Higgs trapped in the wrong planckian vacuum. This requirement either places an upper limit on $H_{\text {inf }}$ and hence on the amplitude of the relic gravity waves one may expect to observe, or asks for the new physics at the lower energy scale which changes the Higgs potential in a way to make the inflationary dynamics safe from falling into the wrong vacuum (e.g. by getting rid of this vacuum). The limit depends on the exact values of the Higgs boson mass $m_{h}$ and the top-quark mass $m_{t}$ as shown on left panel of Fig. 3. This is one insight into the new high energy physics we gain from consideration of the early-time cosmology.

\section{Inflationary models with concave potential and perturbative quantum corrections}

Detailed measurements of matter power spectrum parameters allows one to extract the information about the primordial spectrum of scalar perturbations, parameterised as shown by (7). These observ- 

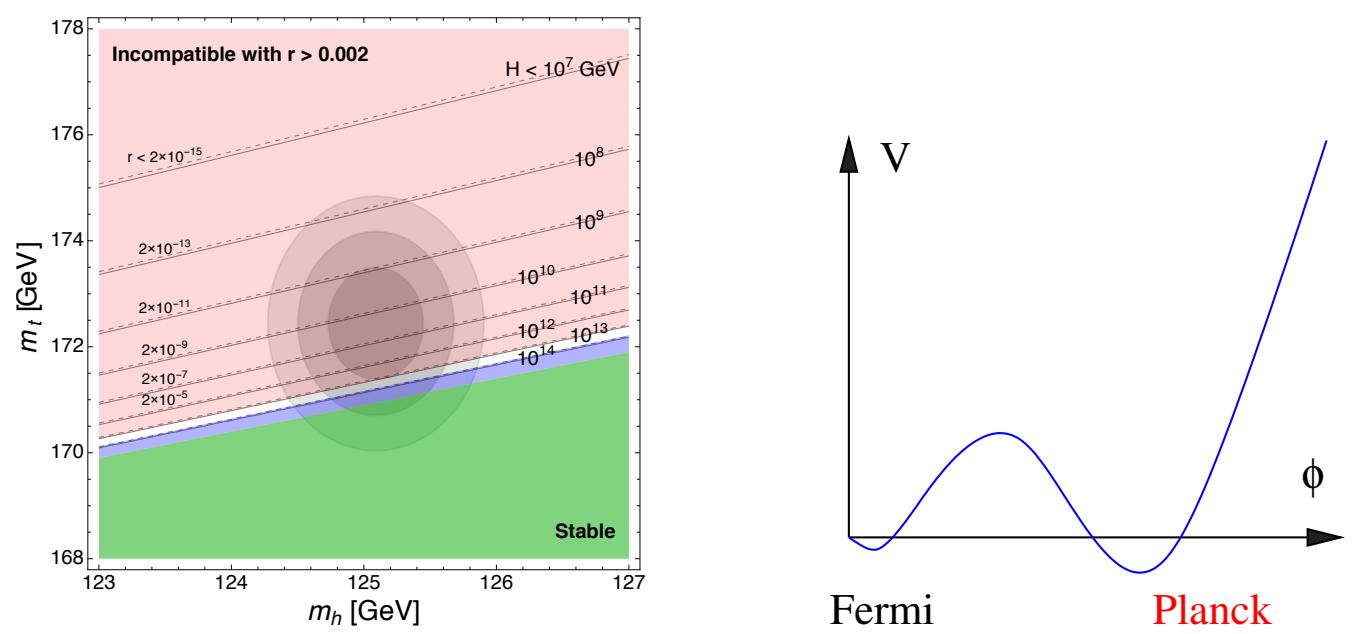

Figure 3. Left panel: Upper limits on the Hubble parameter at inflation and on the tensor-to-scalar ratio $r$ (9) depending on $\left(m_{h}, m_{t}\right)$ [3]. Right panel: a sketch of the SM effective Higgs potential corresponding to the presently measured central values of $\left(m_{h}, m_{t}\right)$.

ables are related to the inflaton potential,

$$
A_{S} \rightarrow \frac{V^{3 / 2}}{V^{\prime}}, \quad n_{S} \rightarrow \frac{V^{\prime \prime}}{V},\left(\frac{V^{\prime}}{V}\right)^{2}, \quad r \equiv \frac{A_{T}}{A_{S}} \rightarrow\left(\frac{V^{\prime}}{V}\right)^{2},
$$

where $A_{T}$ refers to the magnitude of the tensor power spectrum defined analogously to the scalar case (6), (7). The experimental data are normalized to a particular value of $k_{*}$ in (7), which refers to the mode exiting the horizon some $N_{e} \sim 50$ e-foldings before the end of inflation (that means, after the exit the scale factor grows by factor $\mathrm{e}^{N_{e}}$ till the inflation gets terminated). The exact number depends on the post-inflationary history of the Universe. If the inflaton sector is fully determined, and the inflaton coupling to the SM fields, responsible for the subsequent reheating of the Universe, is defined too, then $N_{e}$ is fixed either, as it is obvious from Fig. 2.

So far, no evidences for the gravitational waves have been found, but the amplitude and the tilt of the scalar perturbations are measured with high accuracy, the results are presented in Fig. 4. Note that the most natural and simple models of scalar field with power-law potential, $V(\phi) \propto \phi^{\alpha}$, the quadratic, $V(\phi) \propto \phi^{2}$, and quartic ones, $V(\phi) \propto \phi^{4}$, [7] are excluded (or the former is at least strongly disfavored) by the present cosmological data. The strong upper limit on the gravity waves separates as viable the models with very flat potentials, as clearly seen from the expression for the tensor-to-scalar ratio,

$$
r=\frac{A_{T}}{A_{S}} \propto \frac{\dot{\phi}^{2}}{H^{2} M_{\mathrm{Pl}}^{2}} \propto\left(\frac{V^{\prime}}{V}\right)^{2} \ll 1,
$$

the latter constraint is inherent in the slow-roll approximation guaranteeing the almost exponential expansion during inflation.

Moreover, as a general tendency one can observes with a closer look at Fig. 4, that the presently available data point at the large field inflation models with concave scalar potentials (check for $V(\phi) \propto$ $\phi^{\alpha}$ with $\left.\alpha<1\right)$ as certainly more preferable. This finding seems good for robustness of the inflation 


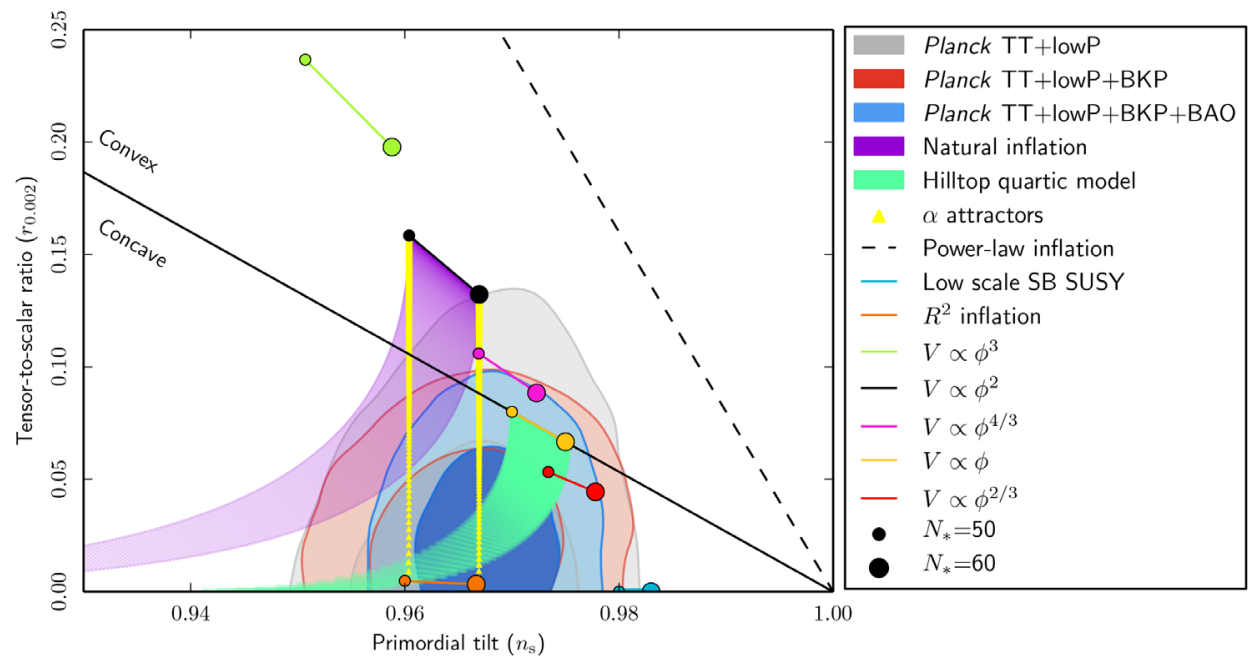

Figure 4. Present cosmological constraints [1] on $\left(r, n_{s}\right)$ and predictions of a set of popular inflationary models. The colored regions are allowed at $65 \%$ and $95 \% \mathrm{CL}$, the model predictions depend on the number of $e$-folding $N_{e}$ remained after the horizon crosssing.

predictions of the cosmological parameters, but seems bad for any other possible low-energy check of the inflaton sector, including the reheating dynamics, which in turn limit the accuracy of the inflation predictions of cosmological parameters.

The problem can be illustrated with an example of exponentially flat potential, typical for many well-developed cosmologically viable models, including very popular $R^{2}$-inflation [4], Higgs-inflation [5] and $\alpha$-attractors [6]. In the former two cases the effective scalar potential at the inflationary stage coincide,

$$
V(\phi)=\frac{3 \mu^{2} M_{\mathrm{P}}^{2}}{4}\left[1-\exp \left(-\sqrt{2 / 3} \phi / M_{\mathrm{P}}\right)\right]^{2},
$$

while in the latter case the numerical factor in the exponent is an arbitrary coefficient (proportional to $\alpha$ !). Here we introduced the reduced Planck mass $M_{\mathrm{P}}^{2} \equiv M_{\mathrm{Pl}}^{2} / 8 \pi$ and dimension-of-mass parameter $\mu$. All the models in the asymptotic of large fields exponentially approach the constant value $V(\phi \rightarrow \infty)$ which defines the amplitude of the tensor perturbations by eq. (8). In both $R^{2}$-inflation and Higgsinflation there is only one model parameter, represented here as $\mu$. And it is fixed by matching to the measurements of the CMB anisotropy $\delta T / T \sim 10^{-4}$ as

$$
\mu \approx 10^{13} \mathrm{GeV} .
$$

In case of $\alpha$-attractors there is some freedom after the matching to CMB because there are two model parameters.

The inflaton potential (10) is plotted in Fig. 5. In Higgs-inflation and $R^{2}$-inflation the postinflationary stage and reheating are fixed by the SM interactions and gravity, respectively, so that both the expansion law and the reheating temperatures are known $[8,9]$. Therefore, for the mode $k_{*}$ used as a normalization in the present cosmological data analyses we know the number of $e$-foldings $N_{e}$ remained from its horizon exit till the end of inflation, and hence the exact value of the field $\phi$ 


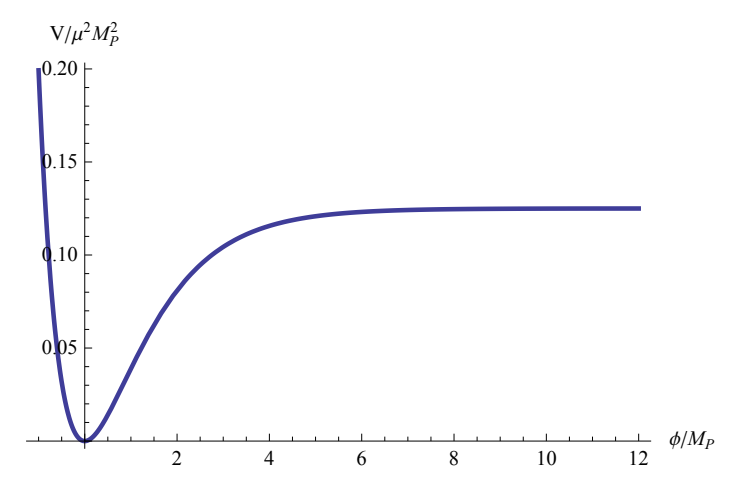

Figure 5. An example of the concave-type flat inflaton potential shared by $R^{2}$-, Higgs- and $\alpha$-attractor inflations. At inflationary stage the inflaton starts with superplanckian fields and slowly rolls along the potential slope mimicking almost constant energy density, so the Universe exhibits (almost) exponential expansion. The slow rolling stops at some point with inflaton field of a subplanckian value and the inflaton starts to oscillate, so the Universe enters a stage of decelerating expansion.

where we probe the scalar potential with the cosmological data, see Figs. 2, 5. Being exponentially flat at this region, the potentials are "exponentially insensitive" to possible quantum corrections [10], protected by the approximate shift-symmetry, $\phi \rightarrow \phi+$ const. It proves the robustness of the inflationary predictions of the cosmological parameters, such as amplitudes and tilts of the scalar and tensor spectra.

However, the scalar potential is obviously non-renormalizable, and there are no symmetries to protect its form at the quantum level. Naturally, close to the origin, the potentials in all the three models take their unique renormalizable polynomial form, saturated by either quadratic or quartic terms. The quantum corrections to these couplings are under control. The corresponding coupling terms may be naively extracted from the original large-field potential (10) and thus related to the small-field dynamics important for cosmology (e.g. reheating) or even direct tests of the inflaton sector like in Ref. [11]. This procedure, unfortunately, is not justified at all, given the fact that to relate the small-field polynomial behavior and large-field exponential behavior of the scalar potential one must cross the intermediate region where neither the renormalizability nor the shift symmetry prevents the potential from getting unknown and hence untamable quantum corrections.

This observations prevents from matching the small-field and large-field parameters of the model. Hence the power of the model predictions are rather limited. In particular, in the Higgs-inflation one cannot predict the cosmological parameters from the measurement of the Higgs boson mass. In $R^{2}$-model, the mass term $\mu$ in (10), which value is fixed from the CMB anisotropy measurements, may not be the same at reheating. Note, that since reheating temperature enters logarithmically to the number of efoldings $N_{e}$, it introduces the corresponding uncertainty into the model predictions of the cosmological parameters. Naturally it is small, however in some cases it may be crucial. For instance, the difference between the predictions of cosmological parameters obtained for Higgs-inflation and $R^{2}$-inflation is quite small and mostly attributed to the difference in reheating temperatures $[12,13]$. The uncertainty associated with nonrenormalizability of the models seems enough to prevent from precise distinguishing between the two.

To cure the situation one must either invoke some symmetry to protect the form of the inflaton potential or to learn how to properly account for quantum corrections from the gravity sector.

\section{Summary}

The present cosmological observations confirm that the Universe was as hot as $2-3 \mathrm{MeV}$ and most probably (much) hotter. The presence of dark matter and the baryon asymmetry of the Universe certainly imply new physics active in the very early Universe, but the cosmology does not point at particular energy scales. Inflation must proceed keeping save the electroweak vacuum, the requirement 
which can suggest the new physics coupled to the SM Higgs sector. At the moment (and probably in the near future), it is difficult to prove, because a new collider is needed to pin down $m_{t}$ and $m_{h}$ and thus settle the matter of where is the large-field minimum of the SM Higgs potential. Finally, matter inhomogeneities of the largest sizes can allow us to probe physics at the highest energies (inflation). However, (most) cosmologically favorable models are nonrenormalizable, which prevents any direct connection between small- and large-field model parameters. We are not (yet) ready to test properly the high energy physics with large-scale cosmological observations, a breakthrough in our understanding of quantum gravity and large-field dynamics is needed to open this possibility.

The work is supported by the RSF grant 14-12-01430.

\section{References}

[1] Ade P.A.R. et al. [Planck Collaboration], Astron. Astrophys. 594, A20 (2016).

[2] Gorbunov D.S. and Rubakov V.A., Introduction to the theory of the early universe: Cosmological perturbations and inflationary theory, (Hackensack, USA: World Scientific, 2011), 489.

[3] W. E. East, J. Kearney, B. Shakya, H. Yoo and K. M. Zurek, [arXiv:1607.00381 [hep-ph]].

[4] Starobinsky A.A., Phys. Lett. B 91, 99 (1980).

[5] Bezrukov F.L. and Shaposhnikov M.E., Phys. Lett. B 659, 703 (2008).

[6] R. Kallosh, A. Linde and D. Roest, Phys. Rev. Lett. 112 (2014) no.1, 011303 doi:10.1103/PhysRevLett.112.011303 [arXiv:1310.3950 [hep-th]].

[7] Linde A.D., Phys. Lett. B 129, 177 (1983).

[8] Bezrukov F., Gorbunov D. and Shaposhnikov M., JCAP 0906, 029 (2009).

[9] Gorbunov D.S. and Panin A.G., Phys. Lett. B 700, 157 (2011).

[10] Bezrukov F.L., Magnin A. and Shaposhnikov M., Phys. Lett. B 67588 (2009).

[11] F. Bezrukov and D. Gorbunov, JHEP 1005 (2010) 010 doi:10.1007/JHEP05(2010)010 [arXiv:0912.0390 [hep-ph]].

[12] Bezrukov F.L. and Gorbunov D.S., Phys. Lett. B 713, 365 (2012).

[13] Gorbunov D. and Tokareva A., JCAP 1312, 021 (2013). 